\title{
CONCEPÇÃO DOS ENFERMEIROS ACERCA DA CAPACITAÇÃO NO CUIDADO À CRIANÇA COM CÂNCER
}

\author{
Daniela Doulavince Amador', Isabelle Pimentel Gomes 2, Simone Elisabeth Duarte Coutinho ${ }^{3}$, Teresa \\ Neumann Alcoforado Costa ${ }^{4}$, Neusa Collet ${ }^{5}$
}

\footnotetext{
${ }^{1}$ Mestranda do Programa de Pós-Graduação em Enfermagem (PPGE) da Universidade Federal da Paraíba (UFPB). Paraíba, Brasil. E-mail: dani_doulavince@yahoo.com.br

${ }^{2}$ Mestranda do PPGE/UFPB. Enfermeira da clínica pediátrica do Hospital Universitário Lauro Wanderley. Paraíba, Brasil. E-mail: enfisabelle@yahoo.com.br

${ }^{3}$ Mestre em Enfermagem. Professora do Departamento de Enfermagem em Saúde Pública e Psiquiatria (DESPP) do Centro de Ciências da Saúde (CCS) da UFPB. Paraíba, Brasil. E-mail: simonedc_3@hotmail.com

${ }^{4}$ Mestre em Enfermagem. Professora do DESPP/CCS/UFPB. Paraíba, Brasil. E-mail: teresalcoforado@ccs.ufpb.br

${ }^{5}$ Doutora em Enfermagem. Professora do DESPP/CCS e do PPGE/UFPB. Paraíba, Brasil. E-mail: neucollet@gmail.com
}

RESUMO: Objetivou-se identificar a concepção dos enfermeiros que trabalham com oncologia pediátrica acerca de como a capacitação e a busca pelo conhecimento influenciam a atuação profissional nessa área. Pesquisa qualitativa, exploratória, com técnica de entrevista semi-estruturada aplicada a seis enfermeiras. Os dados foram submetidos à análise temática a qual convergiu para a seguinte categoria: subsídios que influenciam a produção do cuidado do enfermeiro na oncologia pediátrica. Os resultados ressaltam que a atuação em oncologia pediátrica exige profissionais com responsabilidade, compromisso, preparo adequado e sensibilidade para cuidar da criança. A produção do cuidado tem se fundamentado na vivência dos enfermeiros e na busca individual de conhecimento, demonstrando as dificuldades enfrentadas no processo formativo e a importância da capacitação profissional. Acredita-se que tais achados possam subsidiar a formação de enfermeiros com uma perspectiva problematizadora e capazes de promover uma atenção integral, humanizada e resolutiva para as crianças com diagnóstico de câncer.

DESCRITORES: Competência profissional. Educação em enfermagem. Enfermagem oncológica. Pediatria. Cuidados de enfermagem.

\section{NURSES' CONCEPTIONS ABOUT PEDIATRIC ONCOLOGY CARE TRAINING}

\begin{abstract}
The objective of this study is to identify the conceptions of nurses who work with Pediatric Oncology concerning how their training and the further search for knowledge influences their professional practice. It is a qualitative, exploratory type study with semistructured interviews applied to six nurses. The results were submitted to thematic analysis, which converged to the category, "Subsidies that influence the Pediatric Oncology Nursing Care production". The results show that pediatric oncology performance demands professionals with responsibility, commitment, adequate preparation, and sensitivity to take care of the child at hand. Care production has been grounded in the individual experiences of nurses and individual pursuit of knowledge, demonstrating the difficulties encountered in the training process and the importance of professional training. It is believed that these findings may support nursing training with a problematzing perspective and be able to promote comprehensive, humane, and efficient care for children diagnosed with cancer.
\end{abstract}

DESCRIPTORS: Professional competence. Education nursing. Oncologic nursing. Pediatrics. Nursing care.

\section{LA CONCEPCIÓN DE LOS ENFERMEROS SOBRE LA CAPACITACIÓN EN EL CUIDADO DE LOS NIÑOS CON CÁNCER}

\begin{abstract}
RESUMEN: El objetivo del estudio es identificar la concepción de los enfermeros que trabajan con oncología pediátrica sobre cómo la capacitación en esta área y la búsqueda del conocimiento influencian el trabajo profesional. Se trata de una investigación cualitativa exploratoria, con técnica de entrevista semi-estructurada, aplicada a seis enfermeras. Los datos fueron sometidos al análisis temático el cual converge para la categoría: subsidios que influencian la producción del cuidado del enfermero en la oncología pediátrica. Los resultados muestran que el rendimiento de los profesionales de la oncología pediátrica demandan responsabilidad, compromiso, sensibilidad y preparación adecuada para cuidar del niño. La producción de la atención se ha basado en la experiencia de las enfermeras y la búsqueda del conocimiento, lo que demuestra las dificultades encontradas en el proceso de formación y la importancia de la capacitación profesional. Se cree que estos hallazgos pueden apoyar la formación de enfermeros con una perspectiva problemática y capaz de promover la atención integral, humana y eficaz para los niños diagnosticados con cáncer.
\end{abstract}

DESCRIPTORES: Competencia profesional. Educación en enfermería. Enfermería oncológica. Pediatría. Atención de enfermería. 


\section{INTRODUÇÃO}

O câncer, hoje, é considerado um problema de saúde pública global, responsável por cerca de $13 \%$ de todas as causas de óbito no mundo. Estimase que, em 2020, o número de casos novos anuais seja da ordem de 15 milhões. No Brasil, a mortalidade por câncer representa 13,7\%, ficando atrás apenas das doenças do aparelho circulatório, cujo percentual chegou a $27,9 \%$. As mortes por causas externas aparecem em seguida, com 12,4\%. ${ }^{1}$

O câncer infanto-juvenil (abaixo de 19 anos) é considerado raro, quando comparado com os tumores do adulto, que correspondem entre $2 \%$ e 3\% de todos os tumores malignos. No Brasil, que tem uma população jovem, a mortalidade por câncer em crianças e adolescentes com idade entre 1 e 19 anos correspondeu a $8 \%$ de todos os óbitos em 2005, colocando-se, assim, como a segunda causa de morte nessa faixa etária. Esse perfil de óbitos implica uma relação direta com a organização específica dos serviços de saúde e traz novos desafios para a atenção em oncologia e o Sistema Único de Saúde (SUS). ${ }^{2}$

O cenário da atenção na oncologia apresenta desafios enormes, no que diz respeito ao enfrentamento do problema de forma integral. O desenvolvimento de estratégias para o controle do câncer depende da abordagem a problemas que afetam desde os mecanismos de formulação das políticas de saúde até a mobilização social, a organização e o desenvolvimento das ações e dos serviços de saúde e a geração e a difusão de conhecimentos. ${ }^{3}$

Esses desafios exigem cada vez mais trabalhadores qualificados e aperfeiçoados para lidar com as novas demandas do exercício profissional, direcionadas à realidade epidemiológica do nosso país. No que concerne à oncologia pediátrica, é necessário congregar esforços para uma participação mais efetiva dos profissionais de saúde no diagnóstico precoce, no controle da doença e na melhoria da qualidade da assistência prestada.

Existe uma lacuna considerável na capacitação em oncologia, cuja base é a graduação, já que a maioria dos cursos de Enfermagem, geralmente, não oferece um aprofundamento importante nessa área. ${ }^{4}$ Vale salientar que as Diretrizes Curriculares Nacionais para os cursos de graduação em Enfermagem pregam a formação do enfermeiro generalista. ${ }^{5}$ Todavia, o perfil epidemiológico nacional e mundial deve ser observado, e as devidas adaptações curriculares feitas para que a formação atenda às realidades específicas. As próprias insti- tuições onde os enfermeiros trabalham devem ter o compromisso de proporcionar oportunidades para a formação complementar, necessária ao exercício profissional em área especializada, como é o caso da oncologia pediátrica. Contudo, nem sempre, essas instituições oferecem capacitação e atualização, mesmo sendo essa uma área em constante transformação e que tem demandado dos enfermeiros capacitação permanente a fim de estarem instrumentalizados para o cuidado integral e humanizado.

A incidência de casos de câncer está aumentando e novos tratamentos e mudanças no cuidado estão gerando muitos desafios. ${ }^{1,2,6}$ Mediante esse contexto, a enfermagem tem a responsabilidade de acompanhar o desenvolvimento do conhecimento em oncologia, principalmente pelas investigações científicas, que são as peças-chave para o desenvolvimento do cuidado com o paciente oncológico. ${ }^{7}$

Diante disso, surgiram indagações no que diz respeito à forma como os enfermeiros têm trabalhado essa realidade e que transformações eles têm buscado em direção à melhoria e à integralidade do cuidado em oncologia pediátrica. Esses aspectos suscitaram reflexões acerca da relação conhecimento/atuação do enfermeiro na oncologia pediátrica, a partir da seguinte questão norteadora: Qual a concepção dos enfermeiros que atuam no cuidado a crianças com câncer acerca do preparo profissional e da vivência nessa área?

Considerando-se as muitas facetas que permeiam o universo oncológico e pediátrico, é de grande relevância que o enfermeiro esteja seguro em suas práticas cotidianas e transcenda os limites técnicos ao cuidar da criança. Além disso, este estudo busca reforçar e ampliar a reflexão da Enfermagem no que concerne ao aprimoramento do cuidar, da responsabilidade e da sensibilidade para vivenciar as nuanças do câncer infantil.

Face ao exposto, este estudo objetivou identificar a concepção dos enfermeiros que trabalham com oncologia pediátrica acerca de como a capacitação nessa área e a busca pelo conhecimento influenciam a atuação profissional.

\section{METODOLOGIA}

Com o intuito de buscar entender os fenômenos da prática da enfermagem na oncologia pediátrica, foi utilizada a abordagem qualitativa, do tipo exploratória. Esse método foi escolhido por permitir um aprofundamento do objeto pesquisado com maior flexibilidade na condução da 
pesquisa, a fim de obter familiaridade com o problema, por meio da fala dos entrevistados. ${ }^{8}$

A pesquisa de campo foi realizada no Hospital Napoleão Laureano, localizado na cidade de João Pessoa-PB. A escolha por esse cenário se justifica por se tratar de um Centro de Alta Complexidade em Oncologia, filantrópico, referência no tratamento de câncer no Estado e que vem incorporando novos conhecimentos ao seu atendimento cotidiano, que exigem preparo técnico e científico do seu corpo de funcionários, bem como sensibilidade para lidar com a sua clientela.

A inclusão dos participantes da pesquisa obedeceu aos critérios de ser enfermeiro que atuava ou já tinha atuado no setor de pediatria do hospital em estudo e estar disposto a participar da pesquisa. A amostra, de conveniência foi constituída por seis enfermeiras que atuavam no hospital durante o período de coleta de dados, que ocorreu entre os meses de maio e junho de 2009. O critério de encerramento da coleta dos dados foi o de saturação.

A pesquisa foi pautada nas orientações das normas legais que respaldam as pesquisas que envolvem seres humanos, ${ }^{9}$ como o estabelecido na Resolução n ${ }^{\circ} 196 / 96$, e o projeto foi encaminhado e aprovado pelo Comitê de Ética em Pesquisa do Centro de Ciências da Saúde da Universidade Federal da Paraíba - CCS/UFPB, conforme protocolo $\mathrm{n}^{\mathrm{o}}$ 0124. Além disso, foi obtida a aprovação da direção do Hospital para a realização da mesma, e todos os sujeitos assinaram o Termo de Consentimento Livre e Esclarecido.

A técnica de coleta de dados foi a entrevista semi-estruturada, orientada por questões norteadoras que indagavam qual a influência da formação em enfermagem na atuação prática em oncologia pediátrica e a relação entre a prática e o conhecimento produzido na área. As entrevistas foram gravadas em um aparelho MP3 e transcritas na íntegra. No texto, as entrevistadas foram identificadas pela letra $\mathrm{E}$, numeradas conforme a sequência de realização das entrevistas.

A análise dos dados seguiu os passos da análise temática, ${ }^{8}$ cuja operacionalização segue as etapas de pré-análise, que consiste na seleção dos documentos a serem analisados; na exploração do material, objetivando a compreensão do texto, e na análise final, a partir da identificação dos núcleos de sentido cuja presença ou frequência contribuam para o objetivo analítico visado.

Destarte, a partir dos núcleos de sentido, processou-se o reagrupamento dos temas mais relevantes, permitindo refinar a classificação para a realização da análise final. A partir da classificação e da agregação dos dados, foi construída a categoria empírica: Subsídios que influenciam a produção do cuidado do enfermeiro na oncologia pediátrica, a qual, por sua vez, encontra-se dividida em cinco subcategorias: capacitação do enfermeiro que atua em oncologia pediátrica; a busca pelo conhecimento fundamentando a prática em oncologia pediátrica; a vivência prática dos enfermeiros; o processo educativo dos enfermeiros na instituição e o envolvimento emocional em oncologia pediátrica.

\section{ANÁLISE E DISCUSSÃO DOS RESULTA- DOS}

Na análise e na interpretação dos depoimentos das enfermeiras, organizados na categoria: subsídios que influenciam a produção do cuidado do enfermeiro na oncologia pediátrica, identificaramse as ideias que revelam o exercício profissional cotidiano do enfermeiro, traduzem como este se descortina, seu significado, seus anseios, inseguranças, frustrações e esperança. São aspectos vivenciados na prática que podem construir ou desconstruir um cuidado de qualidade e, portanto, precisam ser refletidos e analisados.

\section{Subsídios que influenciam a produção do cui- dado do enfermeiro na oncologia pediátrica}

O cuidar em oncologia pediátrica é desafiante, pois requer, além de recursos materiais e terapêuticos específicos, uma equipe de saúde atenta para o que permeia o universo infantil. Exige profissionais com responsabilidade, compromisso, preparo adequado e sensibilidade para cuidar da criança. Diante desse quadro, encontramos o profissional enfermeiro, cuja produção do cuidado qualificado é influenciada pelo confronto com a realidade do câncer infantil, que inclui aspectos práticos e emocionais nem sempre presentes. Aliado a isso, existem limitações de ordem institucional, no que diz respeito à falta de uma contínua atualização dos conhecimentos e das experiências que desencadeiam sentimentos como despreparo e impotência.

Os enfermeiros, ao relatar suas experiências no cuidado à criança com câncer, mostraram facetas que, pela convergência das unidades de significado dos seus depoimentos, puderam ser agrupadas em cinco subcategorias: Capacitação do enfermeiro que atua em oncologia pediátrica; A busca pelo conhecimento fundamentando a prática 
em oncologia pediátrica; A vivência prática dos enfermeiros; $\mathrm{O}$ processo educativo dos enfermeiros na instituição e $\mathrm{O}$ envolvimento emocional em oncologia pediátrica.

\section{Capacitação do enfermeiro que atua em oncologia pediátrica}

As altas taxas de morbimortalidade por câncer, em nosso país, a crescente incorporação tecnológica nas ações de controle do câncer, as novas tendências profissionais, as mudanças sociais e governamentais, bem como as novas concepções do processo saúde doença estão exigind o a formação de profissionais com base de conhecimentos que lhes possibilitem organizar e implementar práticas qualificadas, ampliadas e integradas de cuidados à saúde da clientela alvo da oncologia. ${ }^{10}$

Os relatos das enfermeiras acerca do ensino da oncologia, na grade curricular do Curso de Graduação em Enfermagem, demonstram que esse ainda é escasso ou bastante limitado, o que implica dificuldades de atuar na área e de produzir um cuidado ampliado à criança com câncer a partir do ensino da graduação, como relatado a seguir: [...] quando eu me formei eu não paguei [cursei] oncologia prática, só paguei [cursei] oncologia na teoria, a gente sabe que é muito diferente [...] você não se sente preparada [...] tinha dificuldade nos nomes, você não sabe o que é que vem primeiro, a ordem da infusão dos quimioterápicos (E2); [...] eu entrei aqui [serviço de oncologia pediátrica], sem nenhuma informação de como era, o que eu estava fazendo, como era que eu ia fazer [...] para que servia aquela medicação que estava sendo feita (E3).

Os egressos dos cursos de graduação em Enfermagem enfrentam dificuldades relacionadas às novas demandas geradas pela estreita dependência com o mundo do trabalho. A oncologia é uma área muito específica, que não faz parte, na maioria das vezes, do currículo generalista para a formação do enfermeiro.

As constatações da necessidade de formar enfermeiros que respondam às necessidades da população e dos serviços de saúde não são recentes. Em 1987, durante o $1^{0}$ Simpósio Brasileiro sobre Educação em Cancerologia, realizado em Brasília, formou-se a Comissão Nacional para o Ensino de Cancerologia nos Cursos de Graduação em Enfermagem, na qual foi elaborado o documento "Ensino da Cancerologia nos Cursos de Graduação em Enfermagem", objetivando capacitar o futuro enfermeiro para desempenhar ações específicas na área de Enfermagem e forne- cer bases educacionais para o planejamento e a implantação de programa de controle e prevenção de neoplasias prevalentes. ${ }^{11}$

O impacto pelo esforço e pelo compromisso das pessoas e das instituições envolvidas nesse processo não foi o esperado. Após esse documento inicial, foram realizados seminários sobre o Ensino da Cancerologia nos Cursos de Graduação em Enfermagem, que debateram estratégias para operacionalizar a proposta, e alguns avanços foram identificados, entre os quais, a inclusão de experiências práticas em ações de promoção, prevenção e detecção precoce do câncer. Porém, não houve a continuidade necessária para que esses movimentos tivessem prosseguimento. Os avanços ocorridos não são homogêneos em relação à consolidação dos conteúdos de oncologia na formação superior do enfermeiro. ${ }^{12}$

A Lei de Diretrizes e Bases da Educação Nacional (LDB) - Lei n ${ }^{\circ}$ 9.394, de 20 de dezembro de 1996, aponta a adoção de diretrizes curriculares específicas para cada curso. ${ }^{13}$ Os princípios das Diretrizes Curriculares referentes ao ensino de Enfermagem pretendem a formação de um enfermeiro generalista, capaz de reconhecer os problemas/ situações de saúde-doença mais prevalentes no perfil epidemiológico nacional e intervir neles. No entanto, como as Diretrizes preconizam a formação de um enfermeiro generalista, algumas instituições de ensino justificam a exclusão (ou não inclusão) do conteúdo de oncologia por entenderem que este deve ser específico para o ensino em nível de pós-graduação, não demonstrando iniciativas de reelaboração pedagógica, nem contextualizando o perfil do egresso com as necessidades sociais e sanitárias, locais e regionais. ${ }^{14}$

Essas características sentenciam a manutenção de diagnósticos em fases tardias de desenvolvimento da neoplasia em crianças e adolescentes e contribuem para resultados insatisfatórios no tratamento do câncer nesse grupo. A maioria das ações relacionadas ao controle do câncer depende da formação de qualidade dos profissionais de saúde, para que sejam capazes de prevenir, diagnosticar, tratar, evoluir e saber notificar os casos de câncer. $\mathrm{O}$ alcance desse objetivo não é fácil e, por essa razão, há muito que se avançar nessa área.

\section{A busca pelo conhecimento fundamentando a prática em oncologia pediátrica}

A oncologia tem sido amplamente divulgada nos meios de comunicação, devido aos inúmeros 
sucessos terapêuticos e às inovações tecnológicas apresentadas, alicerçadas em profissionais críticos e reflexivos. Existe uma estreita relação entre a competência profissional e a busca pelo conhecimento, uma vez que se vive na era da excelência e da competitividade.

As enfermeiras entrevistadas demonstram que tentam acompanhar os progressos da oncologia, ao ressaltarem que se empenham em adquirir conhecimento para uma melhor atuação na área: [...] hoje eu faço uma especialização em oncologia e a gente paga [cursa] a parte de oncologia pediátrica (E2); [...] sempre que tem curso relacionado à oncologia eu participo, congressos, palestras (E5).

Os depoimentos das entrevistadas evidenciam que os enfermeiros estão aliando a cientificidade ao fazer profissional e não permanecem estáticos ao desenvolvimento da profissão. A possibilidade de contato com os conhecimentos recentes e avançados sobre o câncer proporciona à equipe de enfermagem uma intervenção eficaz, uma atitude crítica, que releva o papel social do pesquisador, comprovando que o pensamento científico não é um sistema acabado, mas uma incerteza motivadora para o cientista. ${ }^{15}$

Os desafios impostos pela realidade oncológica pediátrica precisam ser respondidos pelo enfermeiro, e a resposta de cada novo desafio é que modifica a realidade e o profissional. A elaboração e o desenvolvimento do conhecimento estão ligados ao processo de conscientização, na qual há um mútuo condicionamento entre pensamento e prática, o que permite a aproximação crítica do indivíduo com a realidade. Nessa aproximação, o enfermeiro tem a possibilidade de transcender a esfera da simples apreensão dos conhecimentos para chegar a uma esfera crítica na qual a tomada de decisão passa a ter embasamento e reflexão.

Para desenvolver cientificamente a enfermagem - o que a faz indispensável em todos os âmbitos de atuação profissional em saúde e locais de trabalho - é importante ampliar o processo de construção do conhecimento para além dos limites impostos pela graduação. Significa intensificar e otimizar a realização da pesquisa no exercício profissional como ferramenta capaz de criar diferentes estratégias de soluções de problemas. ${ }^{16}$

A busca pela consolidação do conhecimento de enfermagem é percebida nos depoimentos e indica que há um interesse pelo aperfeiçoamento, mas não pela pesquisa, área de extrema importância, pois envolve uma temática em constante avanço, como a oncologia pediátrica.

\section{A vivência prática dos enfermeiros em onco- logia pediátrica}

A experiência profissional e emocional adquirida pelo enfermeiro no convívio com a rotina, as intercorrências e todo o contexto do câncer infantil impulsionam o desenvolvimento das habilidades requeridas por esse profissional, como evidenciado no depoimento a seguir: [...] A gente aprende mesmo na prática, no dia a dia [...]. Uma boa parte [dos casos] eu já estou acostumada a ver, porque eu estou aqui dia a dia sempre, de segunda a sexta, vendo só isso (E1).

O contexto da prática clínica e as responsabilidades na gestão de situações cotidianas se constituem numa oportunidade distinta e excepcional de prática. Os serviços hospitalares ou centros de saúde representam, para quem aprende, o contexto na qual se vivencia verdadeiramente as situações de aprendizagem que proporcionam a aplicação de saberes previamente adquiridos. Vivenciar a realidade implica na construção da autonomia e do referencial profissional dos enfermeiros promovendo uma reflexão crítica sobre o saber e o fazer. ${ }^{17}$

No entanto, a integração entre a teoria e a prática é uma das principais dificuldades no processo formativo, e a prática clínica permeada pela incerteza, instabilidade e por uma abordagem casuística das situações, muitas vezes não revela a conscientização dos papéis a serem desenvolvidos pelo enfermeiro e das competências requeridas para o seu desempenho. Essa situação implica num quase permanente conflito cognitivo gerador de sentimentos de ansiedade ou de impotência na atuação inicial do enfermeiro. ${ }^{17}$

A dicotomia presente entre teoria e prática pode ser afirmada sob a suposição de que o ensino de enfermagem não prepara suficientemente o enfermeiro para o seu exercício profissional e não atende às exigências atuais do mercado de trabalho. Nessa linha de pensamento, é conveniente alertar que a adequação da escola ao mercado de trabalho precisa ser bem contextualizada, visto que esse mercado é, muitas vezes, caracterizado pela atenção privada e curativa centrada no hospital. ${ }^{13}$ Portanto, não é a prática assistencial tecnicista que se almeja dos enfermeiros cuidadores de crianças com câncer, tampouco a adequação instrumental ao mercado de trabalho do mundo capitalista. Defende-se uma formação que atenda ao perfil epidemiológico populacional. Portanto, nesta discussão, um olhar cuidadoso em relação à magnitude que vem tomando o câncer infantil. 
O processo educativo que se objetiva tem caráter reflexivo, perpassa todos os níveis de atenção e supera o cuidado centrado na doença da criança. A reflexão constante do que-fazer cotidiano do enfermeiro implica a presença curiosa dele frente a sua realidade, requer ação transformadora e busca constante em um processo infindável de invenção/reinvenção. Portanto, reclama reflexão crítica sobre seu ato de conhecer e de produzir o cuidado em oncologia pediátrica.

\section{O processo educativo dos enfermeiros na instituição}

Evidencia-se a oncologia como uma área com inovações constantes, em relação às opções de tratamento, aos protocolos e às maneiras de cuidar. As enfermeiras entrevistadas relatam em seus depoimentos a atuação da instituição frente ao processo educativo: [...] o hospital proporciona treinamento de qualificação, seja na área de quimioterapia, seja na área de urgência [...] tudo que a gente está vendo, aparecendo no curso ajuda muito, porque muitas vezes a gente leva para o curso a dúvida do dia a dia (E1); [...] o que a gente faz aqui [...] é mais uma educação continuada, querendo trazer as inovações para o nosso dia a dia (E6).

Os cursos oferecidos no hospital em estudo, destinam-se a atualizações e a capacitações técnicas, a partir de lacunas identificadas pela própria instituição, de forma não integrada, que corresponde, portanto, a uma visão de educação continuada. Essa forma de organização do trabalho não supera as lacunas identificadas, pois os enfermeiros responsáveis pela operacionalização do cuidado às crianças com câncer, nessa perspectiva de educação continuada, são reduzidos a espectadores e não participam ativamente das propostas de transformação de sua própria realidade.

Embora a educação continuada tenha dado impulsos à melhoria do cuidado em oncologia pediátrica, cumprir com o compromisso de um cuidado responsável e eficaz implica aderir à estratégia de Educação Permanente em Saúde, compreendida como um processo de vinculação de educação e trabalho. Essa se constitui uma ferramenta estratégica para a reconfiguração das práticas de formação, gestão e assistência, para modificar a lógica da formulação de políticas públicas e fortalecer o controle social. ${ }^{3}$

O conhecimento construído e reconstruído ganha significado para aqueles que atuam e é por meio desse processo, de caráter permanente, que são instituídas estratégias/intervenções para melhorar o cuidado de enfermagem. Trilhar esse caminho é fundamental quando a Instituição objetiva um enfermeiro com potencial transformador na prática do trabalho e na interlocução das diversas esferas gestoras e assistenciais, pois a criança com câncer traz consigo o distanciamento da escola e da família, as dificuldades sociais, a falta de apoio psicológico, a rede social fragilizada, entre outros aspectos que necessitam ser discutidos com toda a equipe de saúde.

\section{O envolvimento emocional do enfermeiro em oncologia pediátrica}

A produção do cuidado de enfermagem em oncologia pediátrica vem se especializando e modificando sua compreensão da atenção a ser desenvolvida. A busca por satisfazer às necessidades das crianças, independentemente do seu problema imediato, leva-nos também a uma abordagem do aspecto psicológico incluindo, nesse contexto, o enfrentamento da situação. Para os enfermeiros que atuam com crianças com câncer, lidar com os aspectos psicológicos decorrentes da doença tem sido uma dificuldade, devido à falta de capacitação para o manejo desse problema: [...] na oncologia, geralmente é difícil, e na oncologia pediátrica então se torna mais difícil porque nós não temos esse contato durante a graduação, com a morte, com o lidar com a morte, com a terminalidade, e, na prática da oncologia, a gente lida muito com isso [...] tudo vai para o enfermeiro, então, você absorve aquilo ali (E4).

As enfermeiras evidenciaram que houve déficit na sua formação no processo de ensino do enfrentamento da terminalidade e suas nuanças. Estudos afirmam que não há uma estrutura sistematizada que forneça às cuidadoras mecanismos e instrumentos de suporte e de apoio. ${ }^{18}$ Embora já exista um grande avanço tecnológico no diagnóstico e no tratamento, o câncer ainda se encontra vinculado à ideia de terminalidade, $\mathrm{o}$ que acarreta, nas enfermeiras, sentimentos de angústia, sofrimento e impotência diante da morte. ${ }^{5}$ Muitas vezes, a atitude negativa do profissional em relação ao câncer tem sua origem na experiência limitada de ensino-aprendizagem direcionada a essa temática.

As entrevistadas não referiram sobre a existência de suporte emocional e psicológico da instituição para a qual trabalham. É possível que o enfermeiro lance mão de algumas estratégias para se proteger da ansiedade que as emoções 
suscitadas no dia a dia provocam, mas é crucial o apoio das instituições que cuidam de crianças na oncologia para darem o aporte necessário à equipe de saúde, com o intuito de prevenir, inclusive, doenças ocupacionais.

\section{CONSIDERAÇÕES FINAIS}

As lacunas da formação nos Cursos de Graduação em Enfermagem têm sido um dos obstáculos para a atuação dos enfermeiros no cuidado à criança com câncer, no início da carreira profissional, o que interfere na possibilidade de construção do cuidado integral e humanizado. A produção do cuidado tem se fundamentado na vivência desses enfermeiros e na busca individual de conhecimento, demonstrando as dificuldades enfrentadas pelos egressos do Curso de Graduação em Enfermagem e a importância da capacitação profissional.

A inserção de conteúdos relativos ao câncer que perpassassem as disciplinas que compõem a matriz curricular do Curso de Enfermagem precisa ser devidamente discutida e colocada em escala de prioridade visto que as enfermeiras, neste estudo, evidenciaram a necessidade de reformas curriculares que venham atender às exigências do mercado de trabalho e que favoreçam o desenvolvimento de competências e de habilidades gerais e específicas, objetivando um profissional egresso qualificado, reflexivo e pronto para atuar sobre a realidade epidemiológica e social oncológica.

A fragmentação dos conteúdos ensinados, desvinculados do processo de trabalho, dificulta a formação do enfermeiro crítico-reflexivo, e o conhecimento científico continua desvinculado do mundo prático. O que se almeja é a formação de enfermeiros com uma perspectiva problematizadora e contextualizada da realidade, em um determinado contexto histórico e social, para que eles sejam capazes de recriar suas ações, desvelar as situações limites e desafiadoras e promover uma atenção integral, humanizada e resolutiva para as crianças com diagnóstico de câncer. Nessa perspectiva, o desafio é que o enfermeiro aprenda a aprender, transformando o conhecimento em conduta humana relevante ao seu exercício profissional.

\section{REFERÊNCIAS}

1. Ministério da Saúde (BR). Ações de enfermagem para o controle do câncer: uma proposta de integração ensino-serviço. $3^{\mathrm{a}}$ ed. Rio de Janeiro (RJ): INCA; 2008.
2. Ministério da Saúde (BR). Câncer da criança e adolescente no Brasil: dados dos registros de base populacional e de mortalidade. Rio de Janeiro (RJ): INCA; 2008.

3. Vincent SP. Educação permanente: componente estratégico para a implementação da política nacional de atenção oncológica. Rev Bras Cancerol. 2007 Jan-Mar; 53(1):79-85.

4. Calil AM, Prado CO. Ensino de oncologia na formação do enfermeiro. Rev Bras Enferm. 2010 Jul-Ago; 63(4):671-4.

5. Lopes Neto D, Teixeira E, Vale EG, Cunha FS, Xavier IM, Fernandes JD, et al. A aderência dos cursos de graduação em enfermagem às diretrizes curriculares nacionais. In: Ministério da Saúde (BR). Ministério da Educação. A aderência dos cursos de graduação em enfermagem, medicina e odontologia às diretrizes curriculares nacionais. Brasília (DF): Ministério da Saúde; 2006. p. 31-86.

6. Ministério da Saúde(BR). Estimativa 2010: incidência de câncer no Brasil. Rio de Janeiro (RJ): INCA; 2008.

7. Silveira CS. A pesquisa em enfermagem oncológica no Brasil: uma revisão integrativa. [dissertação]. Ribeirão Preto (SP): Universidade de São Paulo, Escola de Enfermagem de Ribeirão Preto; 2005.

8. Minayo MCS. O desafio do conhecimento: pesquisa qualitativa em saúde. $9^{a}$ ed. São Paulo (SP): Hucitec; 2007.

9. Ministério da Saúde (BR). Conselho Nacional de Saúde, Comissão Nacional de Ética em Pesquisa. Resolução ${ }^{\circ} 196$ de 10 de outubro de 1996: diretrizes e normas regulamentadoras de pesquisa envolvendo seres humanos. Brasília (DF): MS; 1996.

10. Gutierrez MGR. A necessidade de formação de especialistas na área de enfermagem em oncologia [editorial]. Acta Paul Enferm. 2004 JulSet; 17(3):253.

11. Ministério da Saúde (BR). Campanha Nacional de Combate ao Câncer/Sistema Integrado e Regionalizado de Controle do Câncer. Ensino da Cancerologia nos cursos de graduação em Enfermagem. Rio de Janeiro (RJ): MS: 1988.

12. Gutiérrez MGR, De Domenico EBL, Moreira MC, Silva LMG. O ensino da cancerologia na enfermagem no Brasil e a contribuição da Escola Paulista de Enfermagem - Universidade Federal de São Paulo. Texto Contexto Enferm. 2009 Out-Dez; 18(4):705-12.

13. Ito EE, Peres AM, Takahashi RT, Leite MMJ. O ensino de enfermagem e as diretrizes curriculares nacionais: utopia x realidade. Rev Esc Enferm USP. 2006 Out-Dez; 40(4):570-5.

14. Paro D, Paro J, Ferreira DLM. O enfermeiro e o cuidar em oncologia pediátrica. Arq Ciênc Saúde. 2005 Jul-Set; 12(3):151-7. 
15. Moreira MC, Camargo TC, Carvalho V, Figueirêdo $\mathrm{CF}$, Rosa LD, Bolzan MF. A pesquisa na área da enfermagem oncológica: um estudo das publicações em periódicos nacionais. Texto Contexto Enferm. 2006 Out-Dez; 15(4):595-600.

16. Stedile NLR. Ensino de enfermagem: momento privilegiado de construção do conhecimento. Acta Paul Enferm. 2002 Jul-Set; 15(3):79-86.
17. Serra MN. Aprender a ser enfermeiro. Identidade profissional em estudantes de enfermagem. Sísifo. Rev Cienc Educ. 2008 Jan-Abr; 5:69-80.

18. Gargiulo CA, Melo MCSC, Salimena AMO, Bara $\mathrm{VMF}$, Souza IEO. Vivenciando o cotidiano do cuidado na percepção de enfermeiras oncológicas. Texto Contexto Enferm. 2007 Out-Dez; 16(4):696-702. 